\title{
Long non-coding RNA LINC00152/miR-613/CD164 axis regulates cell proliferation, apoptosis, migration and invasion in glioma via PI3K/AKT pathway
}

\author{
L. ZHANG ${ }^{1, *}$, Y. WANG ${ }^{2}$, H. SU ${ }^{2}$ \\ ${ }^{1}$ Departtment of Neurosurgery, Heze Medical College, Heze, Shandong, China; ${ }^{2}$ Department of Neurosurgery, Heze Second People Hospital, \\ Heze, Shandong, China \\ ${ }^{*}$ Correspondence: jiang2001324@126.com
}

Received July 6, 2019 / Accepted September 29, 2019

\begin{abstract}
Malignant glioma is the most frequent primary brain tumor in adults. Accumulated evidence showed that long non-coding RNA (lncRNA) long intergenic noncoding RNA 152 (LINC00152) participated in the progression of glioma, while the regulatory mechanisms remain elusive. Here, the study aimed to clarify the partial molecular mechanism of the lncRNA RNA component of mitochondrial RNA processing endoribonuclease (LINC00152) in the progression of glioma. The level of LINC00152, microRNA-613 (miR-613) and the cluster of differentiation 164 (CD164) were measured by quantitative real-time polymerase chain reaction (qRT-PCR) in glioma tissues and cell lines. Western blot was used to detect the expression of CD164, phosphatidylinositol 3' -kinase (PI3K), phosphorylated PI3K (p-PI3K), protein kinase B (AKT), phosphorylated AKT (p-AKT), matrix metalloproteinase 9 (MMP9), BCL2-Associated X (Bax) and c-Myc. Moreover, flow cytometry was carried out to identify cell apoptosis in vitro. Cell migration and invasion in T98G and LN18 cells were determined via transwell assay. Cell proliferation was analyzed by 3-(4, 5-dimethyl-2-thiazolyl)-2, 5-diphenyl-2-Htetrazolium bromide (MTT) assay. Meanwhile, dual-luciferase reporter and RNA immunoprecipitation (RIP) assay were performed to examine the interrelation between miR-613 and LINC00152 or CD164. The levels of LINC00152 and CD164 were obviously increased while miR-613 was especially decreased in glioma tissues and cell lines. The downregulation of LINC00152 and CD164, as well as the upregulation of miR-613 induced cell apoptosis, repressed viability, migration, and invasion. Furthermore, miR-613 was a target gene of LINC00152, while targeted CD164. The knockdown of LINC00152 promoted the expression of Bax, suppressed the levels of PI3K, p-PI3K, AKT, p-AKT, c-Myc, and MMP9. Interestingly, the downregulation of miR-613 or upregulation of CD164 restored the effect of low-expression of LINC00152 on cell proliferation, apoptosis, migration, invasion and the expression of relative proteins in vitro. Low-expression of LINC00152 modified cell proliferation, apoptosis migration and invasion through LINC00152/miR-613/CD164 axis via PI3K/AKT signaling pathway in glioma, thus providing new therapeutic target in the clinical treatment of glioma.
\end{abstract}

Key words: lncRNA LINC00152, miR-613, CD164, glioma, PI3K/AKT pathway

Malignant glioma is the most frequent primary tumor of the brain in adults [1]. More and more studies uncovered the regulatory mechanism in the progression of glioma, while clinical treatment improved little during the past decades [2]. The potential molecular mechanism is complex and needs further researches.

Long non-coding RNAs (lncRNAs) act as non-coding protein function genes [3] and recently their underlying role in the progression and initiation of tumors have been implied [4], including glioma [5]. For example, aberrant expression of metastasis-associated lung adenocarcinoma transcript 1 (MALAT1) is involved in the poor prognosis of colorectal cancer [6]; taurine upregulated gene 1 (TUG1) regulates the process of glioma via promoting cell apoptosis [7]; the colorectal neoplasia differentially expressed (CRNDE) gene enhances glioma cell proliferation and invasion via mTOR signaling pathway [8]. In addition, RNA component of mitochondrial RNA processing endoribonuclease (RMRP) is deemed as an oncogene through promoting KRAS and SOX9 in lung cancer [9]. Overexpression of RMRP promotes the process of glioma by inducing cell proliferation and invasion [10]. The factor of long non-coding RNA long intergenic noncoding RNA 152 (LINC00152) has been revealed that downregulation of it represses glioma cell proliferation and 
invasion by sponging microRNA-4775 in vitro [11]; it also contributes to glioma progression via targeting miR-107 [12]. The potential role of LINC00152 remains unclear, thus this study aimed to figure out the regulatory mechanisms of LINC00152 in the progression and initiation of tumors.

MicroRNAs (miRNAs) are short small noncoding RNA molecules that have been proved to contribute to the tumorigenesis [13], diagnosis, and prognosis of human cancer [14]. Previous studies have shown that knockdown of microRNA$200 \mathrm{c}$ is related to the cancer stem cells of breast cancer [15]; microRNA-223 promotes cell invasion and metastasis in gastric cancer via regulating suppressor gene of Erythrocyte Membrane Protein Band 4.1 Like 3 (EPB41L3) [16]. Wang et al. suggest that the biological function of the microRNA-29 family is connected with the pathophysiologic changes of cancer cells [17]. Meanwhile, microRNA-613 (miR-613) represses cell proliferation and invasion in bladder cancer by modifying Sphingosine kinase 1 (Sphk1) [18] and induces cell cycle arrest in non-small cell lung cancer by mediating Cell Cycle Dependent Kinase 4 (CDK4) [19]. Whether miR-613 is a target gene of LINC00152, and the regulatory mechanism between them still needs to be researched. Hence, further explored the role of miR-613 in the progression of human glioma was utter requisite.

The cluster of differentiation 164 (CD164) is a glycoprotein and a type I integral transmembrane sialomucin. Over the past decades, studies have proved that CD164 may serve as a signaling receptor that regulates cell proliferation and migration in hematopoietic stem and progenitor cells [20]. Tang et al. show that knockdown of CD164 significantly suppresses cell metastasis of colon cancer as CD164 may work via targeting C-X-C chemokine receptor type 4 (CXCR4) signaling pathway [21]. Earlier evidence suggested that CD164 has an utterly important role in ontogenesis and organogenesis, including tumorigenesis. Hence, it was very important to further research the potential role of CD164 in human glioma and other tumors. Above all, whether CD164 was regulated by miR-613 and LINC00152, and the relationship between miR-613 and CD164 and LINC00152 needed to be highlighted.

Herein, the study focused on the effect of LINC00152, miR-613, and CD164 on the progression of glioma via identifying cell proliferation, apoptosis, migration, and invasion in glioma model cells in vitro and in vivo.

\section{Patients and methods}

Clinical samples and cell culture. 25 glioma tissue samples and 25 adjacent normal brain tissue samples were obtained from Heze Second People Hospital via surgical resection and immediately stored at $-80^{\circ} \mathrm{C}$. All the patients involved in the research did not go through other therapy including radiation and chemotherapy and gave the written informed consent before surgery. Above all, the study was approved by the Ethics Committee of Heze Second People Hospital.
All cell lines the study used including LN229, A172, T98G, and LN18 were purchased from American Tissue Culture Collection (ATCC, Manassas, VA, USA), while control HEB cells were obtained from $\mathrm{Be} \mathrm{Na}$ collection (Beijing, China). All cells were cultured with Dulbecco's modified Eagle medium (DMEM; Gibco, Carlsbad, CA, USA). The medium was mixed with $10 \%$ fetal bovine serum (FBS, Gibco) and antibiotics penicillin and streptomycin (Gibco). Subsequently, cells were incubated at $37^{\circ} \mathrm{C}$, with an atmosphere with $5 \% \mathrm{CO}_{2}$.

Cell transfection. Short hairpin RNA (sh-RNA) targeted LINC00152 (sh-LINC00152), CD164 (sh-CD164) and the negative control (sh-NC); overexpressed LINC00152 (LINC00152), CD164 (CD164) and relative control (pcDNA); miR-613 mimic (miR-613) and its negative control (miR-NC); miR-613 inhibitor (anti-miR-613) and its negative control (anti-NC); luciferase reporter vectors of wildtype (WT-LINC00152) and mutant (MUT-LINC00152) of LINC00152, all of them were synthetized in Genewiz (Guangzhou, China). Subsequently, above plasmids or oligonucleotides were transfected into glioma cell lines by Lipofectamine ${ }^{\text {mix }} 2000$ (Invitrogen, Carlsbad, CA, USA) according to the manufacturer's instructions. Finally, all cells were harvested after incubation for the appropriate time.

Quantitative polymerase chain reaction (qRT-PCR) assay. The total cells were harvested and washed by phosphate buffer saline (PBS, Hyclone, Logan, UT, USA), RNA was extracted using Trizol reagent (Invitrogen). PrimeScript ${ }^{\mathrm{Tm}}$ RT reagent Kit with gDNA Eraser (Takara, Dalian, China) was applied to reverse transcription of RNA into cDNA. Subsequently, the quantitative real-time polymerase chain reaction (qRT-PCR) was carried out via TB Green ${ }^{\circledR}$ Premix Ex Taq ${ }^{\text {Tw }}$ II (Tli RNaseH Plus) (Takara) in accordance with the manufacturer's protocol. Mixed solutions could react and the unique signals were collected by Thermal Cycler Dice $^{\mathrm{mt}}$ Real Time System III (Takara). Special primers were as follows: miR-613: (forward: 5'-TGGAATGTAAAGAAGT-3', reverse: 5'-ATCCAGTGCAGGGTCCGAGG-3'); CD164 (forward: 5'-TGAGCCCTGAACACCAGAGAG-3', reverse, 5'-AAAGCCAGATGAGCGCTTCTA-3'); LINC00152 (forward: CTCCAGCACCTCTACCTGTTG, reverse, GGACAAGGGATTAAGACACACA); GAPDH (forward: 5'-ACTTTGTGAAGCTCATTTCCTGGTA-3', reverse: 5'-GTGGTTTGAGGGCTCTTACTCCTT-3').

Western blot assay. The corresponding vector was transfected into glioma cells, the total proteins were harvested by using lysis buffer (Millipore, Bedford, MA, USA) and incubated for $30 \mathrm{~min}$ on the ice. Subsequently, the BCA Protein Assay Kit (Beyotime, Shanghai, China) was adapted to measure protein concentrations. The total protein was denatured by placing in boiling water for $10 \mathrm{~min}$. Afterward, electrophoresis was carried out to separate proteins by using SDS-polyacrylamide gel and the proteins were transfected onto polyvinylidene fluoride (PVDF) membrane (Millipore). $5 \%(\mathrm{w} / \mathrm{v})$ bovine serum albumin (BSA, Amytet Scien- 
tific, Wuhan, China) was used to block PVDF membranes for $2 \mathrm{~h}$, and then membranes were incubated with special primary antibody overnight at $4{ }^{\circ} \mathrm{C}$. Subsequently, PVDF membranes were washed with Tris Buffered saline Tween (TBST, BioTNT, Shanghai, China) 4 times, for 5 minutes. Thereafter, PVDF membranes were incubated with corresponding secondary antibody for $40 \mathrm{~min}$. No-bound antibody on the surface of membranes was washed off by TBST (BioTNT). Finally, SuperSignal Chemiluminescent Substrates (Thermo Fisher Scientific, Rockford, IL, USA) was used to analyze the active signals utilizing Image Lab software (Bio-Rad, Hercules, CA, USA). Special antibodies were as below: CD164 (catalog no. RF5790 and AF3118) were obtained from R\&D Systems (MN, USA); protein kinase B (AKT, catalog no. SAB4500799), phosphorylated AKT (p-AKT, catalog no. SAB4503853), phosphatidylinositol 3'-kinase (PI3K, catalog no. GW21071), phosphorylated PI3K (p-PI3K, catalog no. SAB4502195) and BCL2-Associated X (Bax, catalog no. B8429) were purchased from SigmaAldrich (St. Louis, MO, USA); c-Myc (catalog no. ab32072), matrix metalloproteinase 9 (MMP9, catalog no. 38898) and GAPDH (catalog no. ab8245) were acquired from Abcam (Cambridge, MA, USA).

3-(4,5-dimethylthiazol-2-yl)-2,5-diphenyl-2H-tetrazol3-ium bromide (MTT) assay for cell proliferation. T98G and LN18 cells were seeded into 96-well plates (BD Biosciences, San Jose, CA, USA) at a density of $1 \times 10^{4} / 200 \mu \mathrm{l} /$ well and incubated for $24 \mathrm{~h}$, in three repetitions of per sample. Then $0.5 \mathrm{mg} / \mathrm{ml}$ MTT (MedChemExpress, NJ, USA) was added into each well and cells were cultured for $4 \mathrm{~h}$. Afterward, the supernatant was discarded and $100 \mu$ of dimethyl sulfoxide (DMSO, Solarbio, Beijing, China) was added into each well. As the endpoint, the OD value was analyzed via microplate reader (Bio-Rad) at $490 \mathrm{~nm}$.

Flow cytometry for cell apoptosis. Cell apoptosis was measured by the Annexin V-fluorescein isothiocyanate/Propidium Iodide apoptosis detection kit (Annexin V-FITC/ PI; BD Biosciences) via flow cytometry. Firstly, T98G and LN18 cells were transfected with sh-NC, sh-LINC00152, sh-CD164, miR-NC, miR-613, sh-LINC00152 + pcDNA or sh-LINC00152 + CD164 and cultured for 24 hours at $37^{\circ} \mathrm{C}$ with $5 \% \mathrm{CO}_{2}$. Subsequently, cells were resuspended with binding buffer, and Annexin V-FITC/PI was employed to stain cells according to protocol. Finally, apoptosis signals were measured via FACSCanto II (BD Biosciences).

Transwell assay. Cell migration and invasion were measured by transwell assay. Firstly, cells were seeded into the upper chamber $(8 \mu \mathrm{m}$ pore; Corning Inc., Corning, NY, USA) at a density of $\sim 8 \times 10^{5}$. Subsequently, a complete medium was added into the lower chamber and incubated for $24 \mathrm{~h}$ at $37^{\circ} \mathrm{C}$ with $5 \% \mathrm{CO}_{2}$. Then glioma cells were stained with crystal violet (Sigma-Aldrich), and observed and counted under an inverted microscope. Besides, the upper chamber surface was coated with Matrigel (Corning) prior to the assay of cell invasion via transwell.
Dual-luciferase reporter assay. The binding sites between miR-613 and LINC00152 or CD164 were predicted by StarBase 2.0. The wildtype (WT-LINC00152) and mutant (MUT-LINC00152) of LINC00152 containing binding sites of miR-613, as well as the wildtype (CD164 3'UTR-WT) and mutant (CD164 3'UTR-MUT) were constructed. Subsequently, T98G and LN18 cells were co-transfected with one of them and Renilla plasmid, respectively. As the endpoint, the luciferase activity was captured by using the Dual-Luciferase $^{\oplus}$ Reporter Assay System (Promega, Madison, WI, USA) and identified via Varioskan Flash (Thermo Fisher Scientific) according to protocol.

RNA immunoprecipitation (RIP) assay. RIP assay was conducted to further verify the interaction between miR-613 and LINC00152 or CD164 by the Imprint RNA immunoprecipitation kit (Sigma-Aldrich) according to the manufacturer's instruction. Firstly, glioma cells were transfected with miR-613 mimic (miR-613) or control (miR-NC) and incubated for $48 \mathrm{~h}$. Subsequently, cells were lysed via RIP-buffer and then incubated with magnetic beads covered with Argonaute-2 (AGO2; Abcam) or immunoglobulin G (IgG; Abcam). Finally, unbound material was washed away and total RNA was harvested. The enrichment of LINC00152 and CD164 was examined by qRT-PCR.

Animal experiment. BALB/c male mice were obtained from Shanghai SLAC Laboratory Animal Co., Ltd. (Shanghai, China; $\mathrm{n}=6$ per group) and kept under non-pathogen conditions with 12 hours light/dark cycle and water and food ad libitum. Furthermore, the experiment was approved by the Animal Care and Use Committee of Heze Second People Hospital. T98G cells transfected with sh-LINC00152 or sh-NC were injected into nude mice at the left flank subcutaneously. Then tumor length (L) and width (W) were measured per $7 \mathrm{~d}$ for 4 times, and tumor volume $(\mathrm{V})$ was counted as follows: $\mathrm{V}=\mathrm{L} \times \mathrm{W}^{2} / 2$. After injection for $28 \mathrm{~d}$, all mice were sacrificed; tumor weights were measured and analyzed by GraphPad Prism 7 (GraphPad Inc., La Jolla, CA, USA).

Statistical analysis. All the data were exhibited as means \pm standard deviation (means \pm SD) for three independent experiments and Student's $t$ test was carried out to examine the difference between two groups. Meanwhile, the one-way analysis of variance (ANOVA) was used for multiple groups. Differences were considered statistically significant at $\mathrm{p}<0.05$.

\section{Results}

The expressions of LINC00152 and CD164 were strikingly increased in glioma tissues and cell lines. In order to identify the biological roles of LINC00152 and CD164 in glioma, the levels of LINC00152 and CD164 were detected significantly increased in glioma tissues (Figures 1A, 1B). The expression of CD164 was also detected by western blot; the results showed that LINC00152 and CD164 were significantly increased in glioma cells (Figure 1C). Moreover, 
A

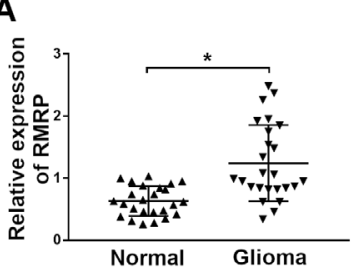

D

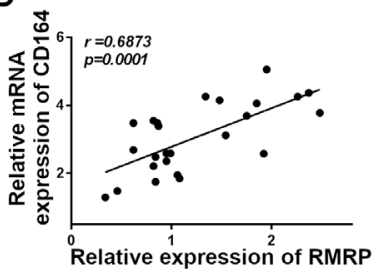

B

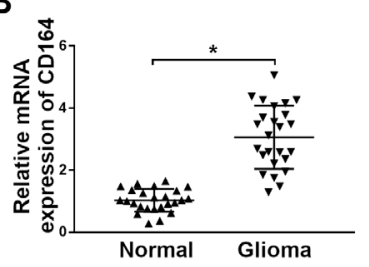

E

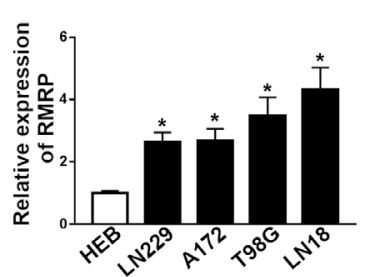

C

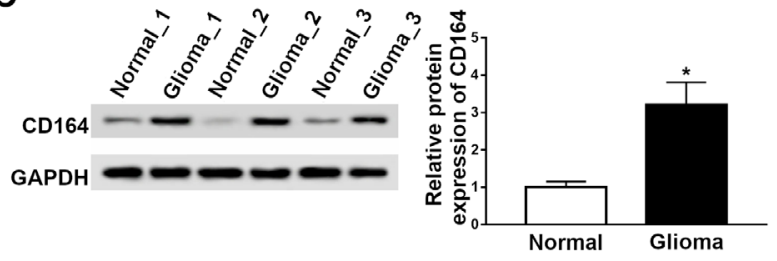

F

G

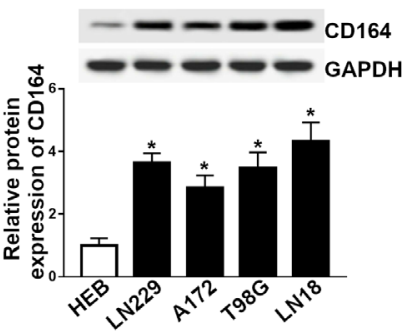

Figure 1. The expressions of LINC00152 and CD164 were strikingly increased in glioma tissues and cell lines. A, B) The levels of LINC00152 and CD164 were measured by qRT-PCR. C) The expression of CD164 was also identified by western blot in glioma tissues compared with normal tissues. D) qRTPCR was carried out to analyze the correlation between LINC00152 and CD164 in human glioma tissues. E, F) qRT-PCR was administrated to detect the level of LINC00152 and CD164 in glioma cell lines. G) The expression of CD164 was also measured by western blot. ${ }^{*} \mathbf{p}<0.05$

A

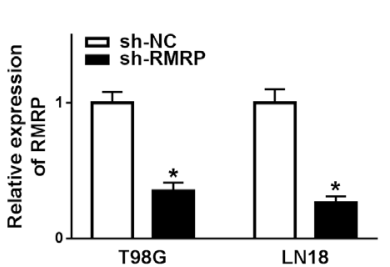

B

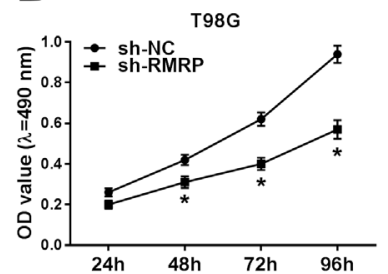

C

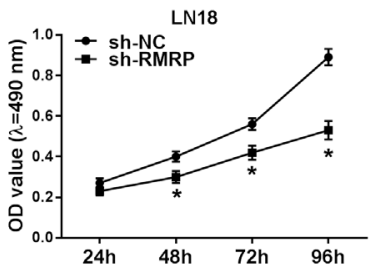

D

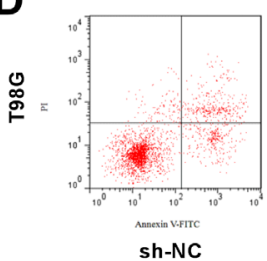

E

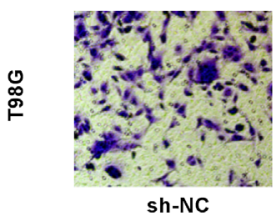

$\mathbf{F}$

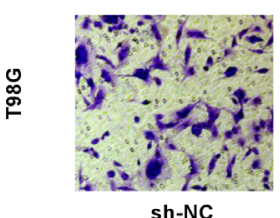

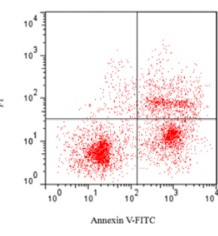

sh-RMRP

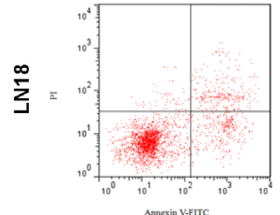

sh-NC
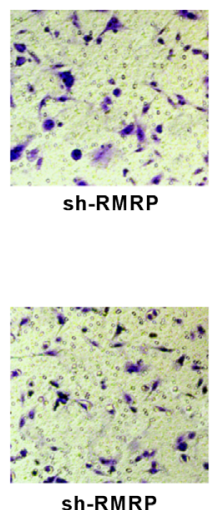
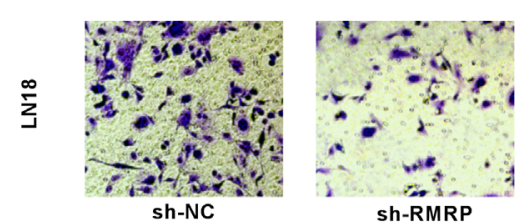

sh-RMRP

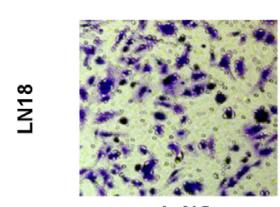

sh-NC

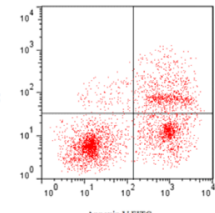

sh-RMRP
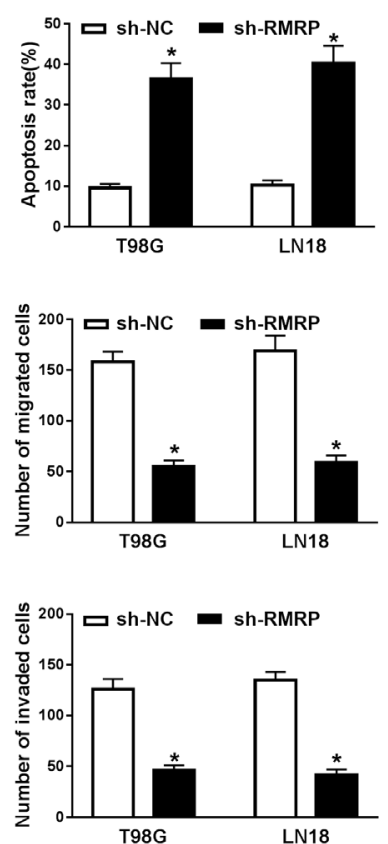

Figure 2. The knockdown of LINC00152 enhanced cell apoptosis and suppressed proliferation, migration, and invasion in vitro. A-G) T98G and LN18 cells were transfected with sh-LINC00152 or sh-NC. A) The level of LINC00152 was detected by qRT-PCR. B, C) MTT assay was performed to identify cell proliferation in glioma cell lines. D) Cell apoptosis was measured using flow cytometry. C, D) Transwell assay was conducted to evaluate cell migration and invasion in vitro. ${ }^{*} \mathrm{p}<0.05$ 
the expression of LINC00152 was positively correlated with CD164 (Figure 1D). Furthermore, the expression of LINC00152 and CD164 were improved in glioma cell lines (LN229, A172, T98G, and LN18) (Figures 1E, 1F). Western blot results showed that CD164 was also increased in glioma cell lines (Figure 1G). The above evidence proved that the levels of LINC00152 and CD164 were obviously promoted in glioma tissues and cell lines, exhibiting the potential role in the progression of glioma.

The knockdown of LINC00152 enhanced cell apoptosis and suppressed cell proliferation, migration, and invasion in vitro. To further investigate the role of LINC00152 in the progression of glioma, T98G and LN18 cells with LINC00152 knockdown were constructed through transfection with sh-LINC00152 or sh-NC for $48 \mathrm{~h}$. The level of LINC00152 was notably repressed in glioma cell lines (Figure 2A). Meanwhile, the repression of cell proliferation was measured by MTT in glioma cell lines (Figures 2B, 2C). Cell apoptosis was increased in T98G and LN18 cells (Figure 2D). In addition, transwell analysis uncovered that the glioma cell migration and invasion were especially suppressed by the knockdown of LINC00152 in vitro (Figures 2E, 2F). In brief, the assay suggested that the downregulation of LINC00152 promoted cell apoptosis and curbed proliferation, migration, and invasion in glioma cell lines.

The downregulation of CD164 induced cell apoptosis and blocked proliferation, migration, and invasion in glioma cells. In order to identify the potential role of CD164 in glioma, we transfected sh-CD164 or sh-NC into glioma cells. The expression of CD164 was particularly repressed (Figures 3A, 3B). Additionally, cell proliferation was significantly decreased in glioma cell lines (Figures 3C, 3D), while cell apoptosis was notably elevated in vitro (Figure 3E). In addition, transwell assay results revealed that cell migration and invasion were obviously hindered by knockdown of CD164 in T98G and LN18 cells (Figures 3F, 3G). In conclusion, enhanced glioma cell apoptosis, hampered proliferation, migration, and invasion were mediated by the knockdown of CD164 in vitro.

The upregulation of CD164 reversed the effect of low-expression of LINC00152 on cell proliferation, apoptosis, migration, and invasion in glioma cells. To analyze the relationship between LINC00152 and CD164, T98G and LN18 cells were transfected with sh-NC,
A

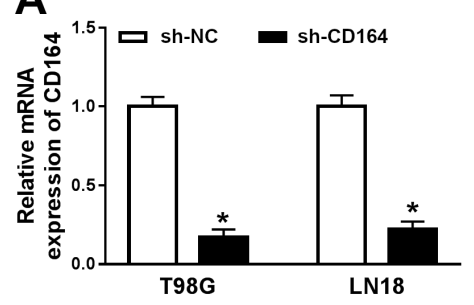

C

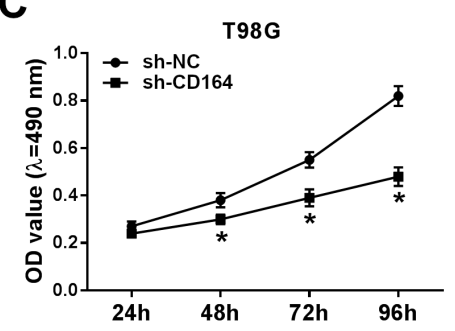

$\mathbf{F}$

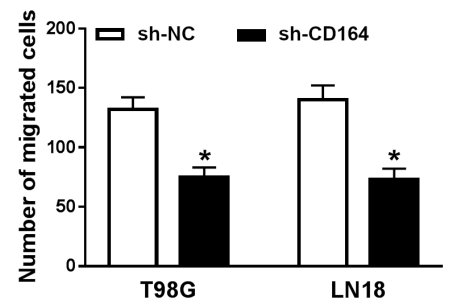

B

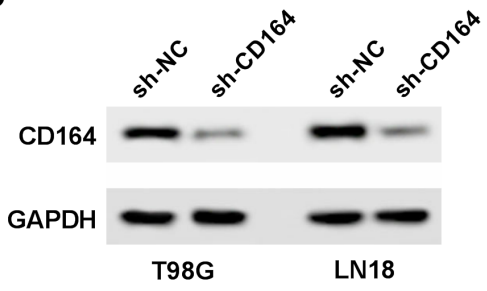

D

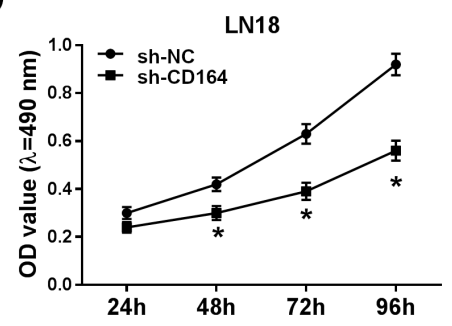

G

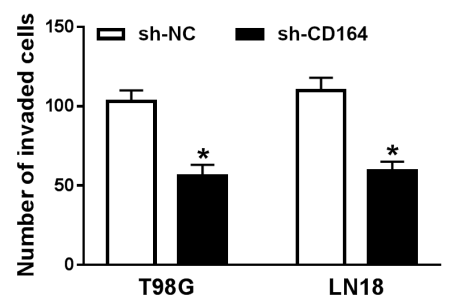

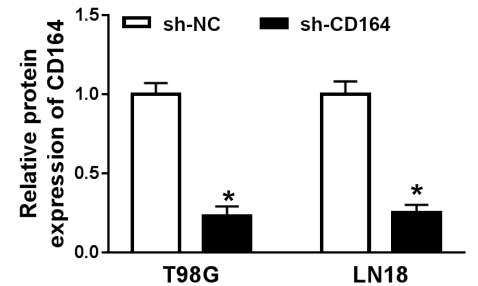

E

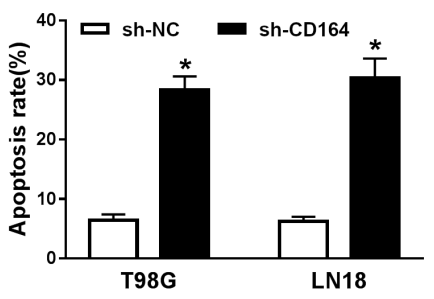

Figure 3. The downregulation of CD164 induced cell apoptosis and blocked proliferation, migration, and invasion in glioma cell lines. A-G) LINC00152 knockdown vector (sh-LINC00152) and relative control were transfected into glioma cell lines. A, B) The level of CD164 was measured by qRT-PCR and western blot, respectively. C, D) Cell proliferation was examined by MTT assay in glioma cell lines. E) Flow cytometry was conducted to analyze glioma cell apoptosis in vitro. F, G) Cell migration and invasion were detected via transwell assay in glioma cell lines. ${ }^{*} \mathrm{p}<0.05$ 
sh-LINC00152, pcDNA or LINC00152, respectively. qRT-PCR and western blot assay showed that the expression of CD164 was notably repressed by sh-LINC00152 while elevated by LINC00152 (Figures 4A-4D). Then, T98G and LN18 cells were transfected with sh-NC, sh-LINC00152, sh-LINC00152 + pcDNA or sh-LINC00152 + CD164 and data proved that overexpression of CD164 restored the effect of knockdown of LINC00152 on cell proliferation (Figures 4E, 4F). The effect of sh-LINC00152 on glioma cell apoptosis (Figures 4G, 4H) was also reversed by CD164 (Figures 4I, 4J). In addition, CD164 as well as regained the effect of sh-LINC00152 on cell invasion in glioma cells (Figures 4K, 4L). From the above, overexpression of CD164 partially reversed the effect of LINC00152 low-expression on cell proliferation, apoptosis, migration, and invasion in vitro.

LINC00152 regulated the expression of CD164 by sponging miR-613. Predicted binding sites of miR-613 and LINC00152 or CD164 utilizing starBase2.0 were exhibited (Figure 5A). Luciferase activity was notably decreased in the wildtype groups (WT-LINC00152 and CD164
3'UTR-WT), while luciferase activity in the mutant groups (MUT-LINC00152 and CD164 3'UTR-MUT) had no particular difference (Figures 5B-5E). To investigate the interrelation of miR-613 and LINC00152 or CD164, T98G and LN18 cells were transfected with miR-NC, miR-613, miR-613 + pcDNA or miR-613 + LINC00152. Western blot assay indicated that the expression of CD164 was significantly inhibited by miR-613 mimic, while the inhibitory effect was reversed by overexpression of LINC00152 in vitro (Figures 5F, 5G). Furthermore, the level of miR-613 was especially decreased in glioma tissues and cell lines (Figures 5H, 5I). The negative correlation between the expression of miR-613 and LINC00152 in glioma tissues was detected by qRT-PCR (Figure 5J). In addition, the efficiency of miR-613 mimic in enhancing miR-613 level was verified, and the results were shown in Figure 5K. Subsequently, the role of miR-613 mimic on cell proliferation was detected using MTT assay, we found that upregulation of miR-613 could distinctly hinder cell proliferation in glioma, whereas this inhibitory effect of miR-613 mimic on cell proliferation
A

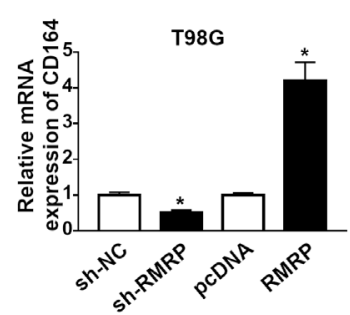

$\mathrm{E}$

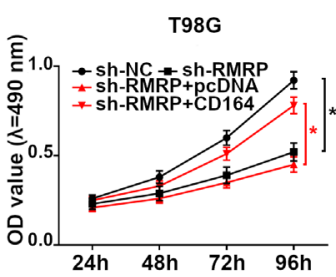

B
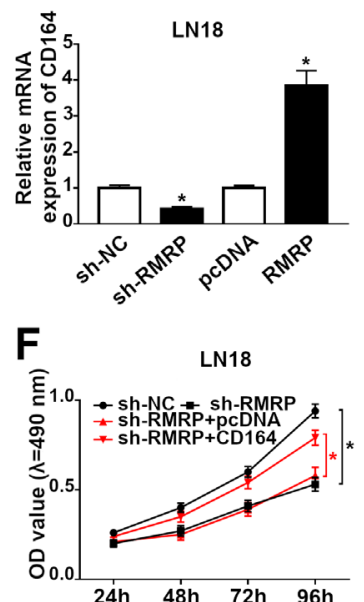

C

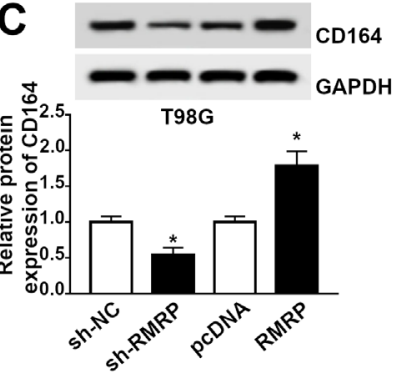

G
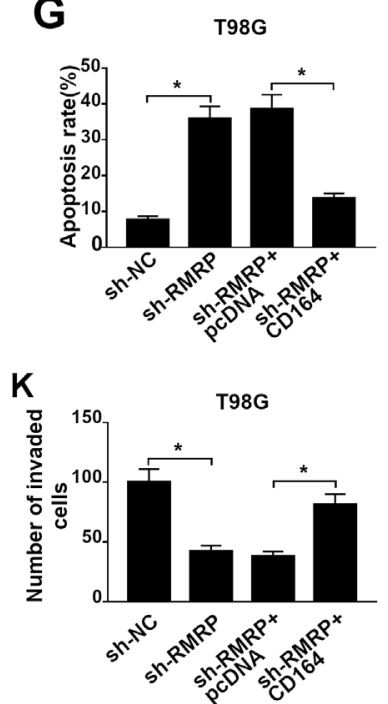

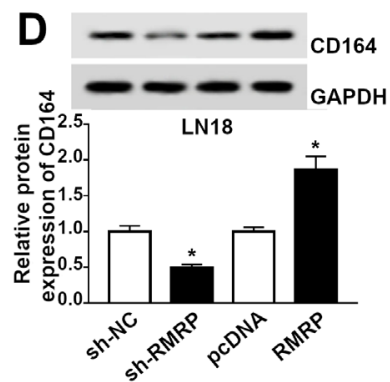

H
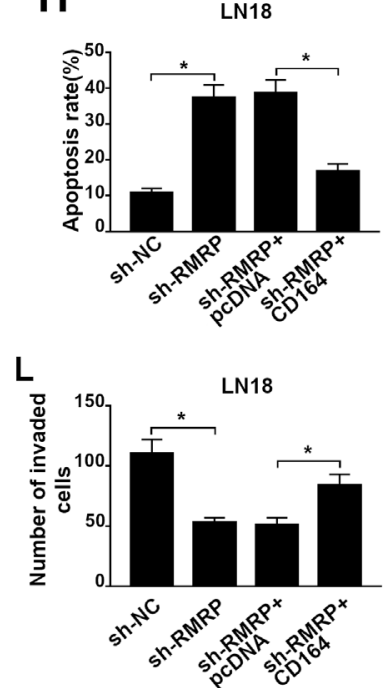

Figure 4. The upregulation of CD164 reversed the effect of low-expression of LINC00152 on cell proliferation, apoptosis, migration, and invasion in glioma cell lines. A-D) T98G and LN18 cells were transfected with sh-NC, sh-LINC00152, pcDNA or LINC00152, respectively and the level of CD164 was examined by qRT-PCR and western blot in glioma cell lines. E-L) After transfection with sh-NC, sh-LINC00152, sh-LINC00152 + pcDNA, and sh-LINC00152 + CD164 into T98G and LN18 cells, severally. E, F) Proliferation of glioma cells was determined by MTT assay in vitro. G, H) Flow cytometry was employed to assess cell apoptosis in glioma cell lines. I-L) Transwell assay was implemented to determine cell migration and invasion of glioma cell lines in vitro. ${ }^{*} \mathrm{p}<0.05$. 

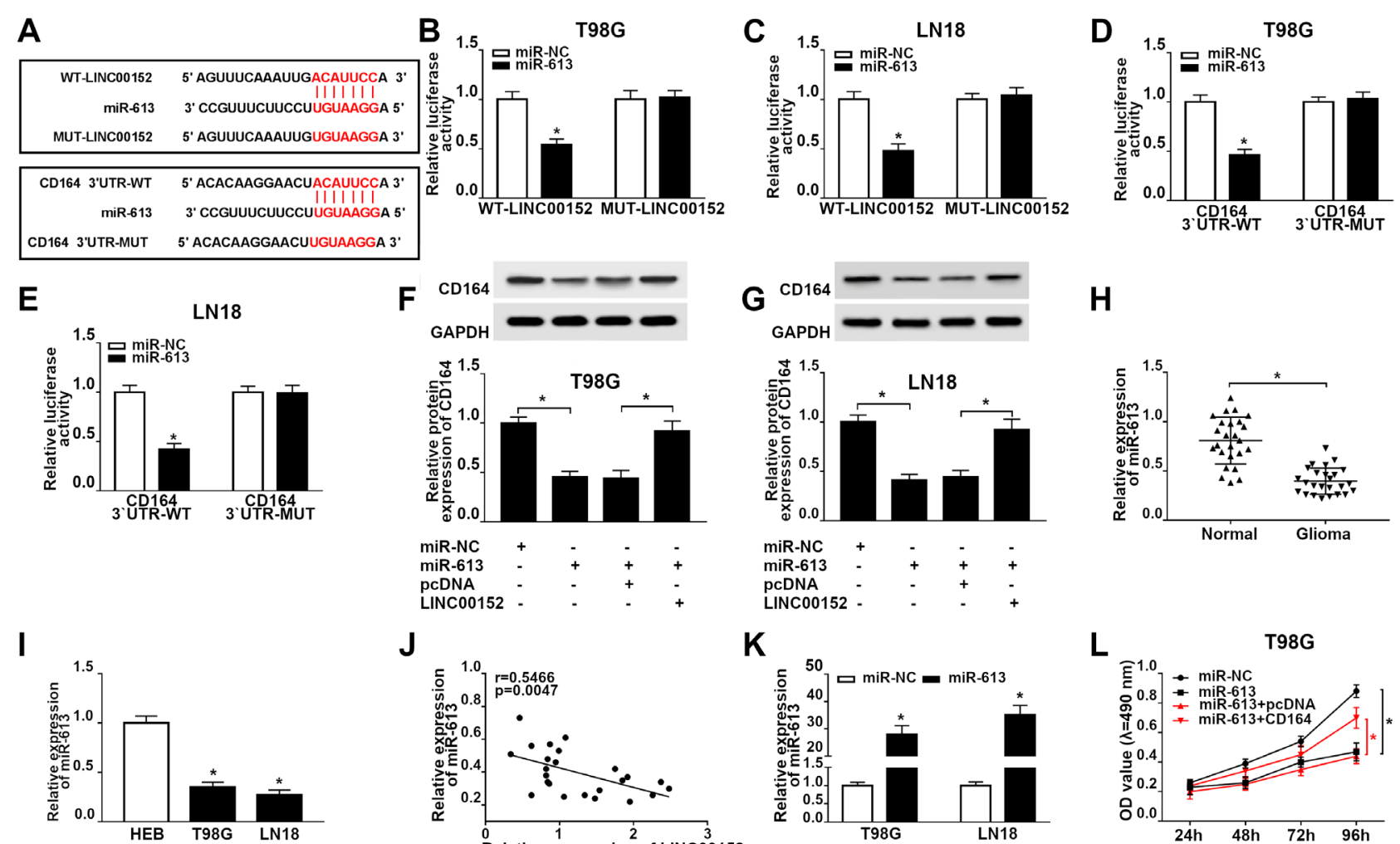

$J$

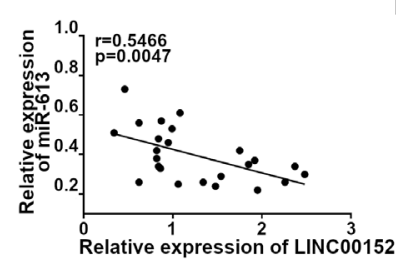

K
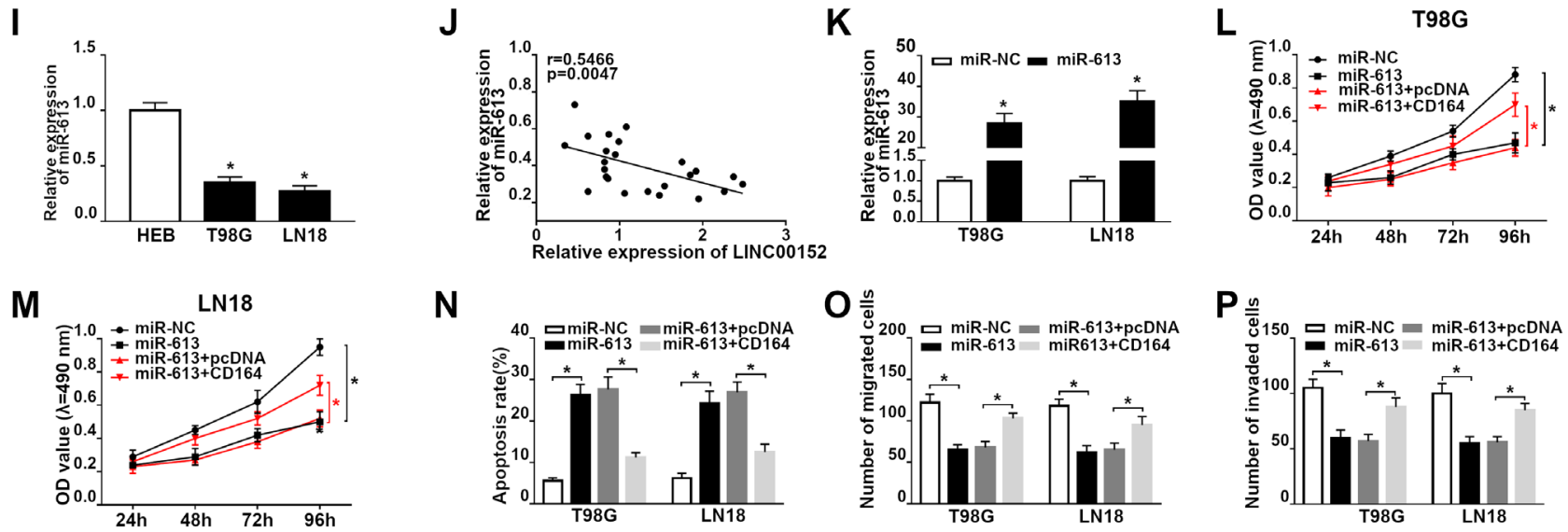

Figure 5. LINC00152 regulated the expression of CD164 by sponging miR-613. A) The binding sites between miR-613 and LINC00152 or CD164 were predicted by starBase 2.0. B-E) Relative vectors were co-transfected with the Renilla vector into T98G and LN18 cells, respectively. Dual-luciferase reporter assay was administrated to analyze the interrelation between miR-613 and LINC00152 or CD164. F, G) miR-NC, miR-613, miR-613 + pcDNA or miR-613 + LINC00152 was introduced into glioma cell lines, severally, and the expression of CD164 was measured via western blot. H, I) qRT-PCR was carried out to determine the level of miR-613 in glioma tissues and cell lines. J) The correlation between LINC00152 and miR-613 was detected by qRT-PCR. K, L) MTT assay was used to identify cell proliferation in glioma cell lines. M) Glioma cell apoptosis was measured utilizing flow cytometry. $\mathrm{N}, \mathrm{O})$ Transwell assay was performed to examine glioma cell migration and invasion in vitro. ${ }^{*} \mathrm{p}<0.05$

was rescued by overexpression of CD164 (Figures 5L, 5M). Simultaneously, CD164 overexpression overturned the acceleratory effect of miR-613 mimic on cell apoptosis in T98G and LN18 cells (Figure 5N). Furthermore, glioma cell migration and invasion were specially curbed by upregulation of miR-613 in vitro, while these effects of miR-613 mimic on cell migration and invasion were abrogated by CD164 introduction (Figures 5O, 5P). In brief, LINC00152 regulated cell proliferation, apoptosis, migration, and invasion by sponging miR-613 through LINC00152/ miR-613/ CD164 axis in the progression of glioma.

LINC00152 regulated the PI3K/AKT pathway by mediating CD164 and miR-613. To further investigate the potential role of LINC00152, miR-613 and CD164 in the progression of glioma, T98G and LN18 cells were transfected with miR-NC, sh-LINC00152, sh-LINC00152 + anti-NC, sh-LINC00152 + anti-miR-613, sh-LINC00152 + pcDNA or sh-LINC00152 + CD164 in vitro, and the protein expression of p-PI3K, PI3K, p-AKT, AKT, c-Myc, Bax and MMP9 were examined by western blot. The results revealed that knockdown of LINC00152 promoted the expression of Bax, blocked p-PI3K, p-AKT, c-Myc, and MMP9, while either downregulation of miR-613 or overexpression of CD164 reversed the effect of sh-LINC00152 on the expression of p-PI3K, p-AKT, c-Myc, Bax, and MMP9 in T98G and LN18 cells (Figures 6A, 6B). Furthermore, we also discovered that PI3K inhibitor could aggravate the role of CD164 detention on repressing cell proliferation in vitro (Supplementary figures S1A, S1B). 

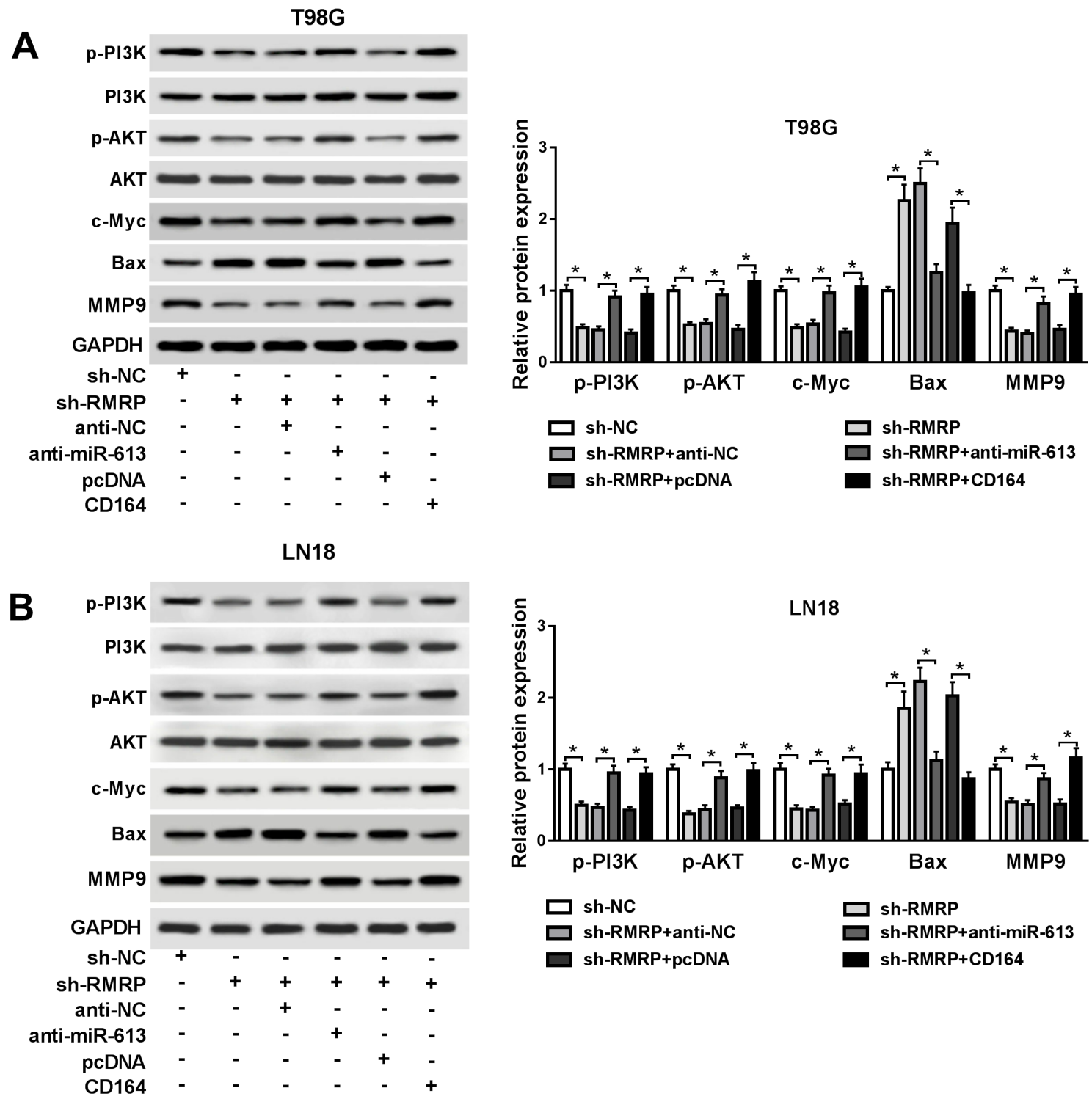

Figure 6. LINC00152 regulated the PI3K/AKT pathway by affecting CD164 and miR-613. A, B) T98G and LN18 cells were transfected with sh-NC, sh-LINC00152, sh-LINC00152 + anti-NC, sh-LINC00152 + anti-miR-613, sh-LINC00152 + pcDNA, sh-LINC00152 + CD164 into T98G and LN18 cells, respectively. The expressions of c-Myc, Bax and MMP9, PI3K, AKT and relative phosphorylation (p-PI3K and p-AKT) were detected by western blot in vitro. ${ }^{*} \mathrm{p}<0.05$

Evidence proved that LINC00152 promoted apoptosisrelative protein expression of Bax, hindered oncogene of c-Myc and invasion-relative protein MMP9. Above all, LINC00152 regulated the PI3K/AKT pathway by mediating $\mathrm{CD} 164$ and miR-613 in vitro.

Knockdown of LINC00152 repressed tumorigenesis in vivo. After injection with a low-expression vector of LINC00152 in nude mice, the tumor volumes and weights were sharply repressed in the sh-LINC00152 group compared with the sh-NC group in vivo (Figures 7A, 7B). The level of LINC00152 was particularly decreased while miR-613 expression was notably increased after injection (Figure 7C). What's more, knockdown of LINC00152 enhanced the expression of Bax, while suppressed the expression of p-PI3K, p-AKT, $\mathrm{c}-\mathrm{Myc}$, and MMP9 (Figure 7D). These findings meant that LINC00152 detention could block the growth of xenograft tumors.

\section{Discussion}

Glioma is the most common primary tumor of the brain and spinal cord [22]. The major gliomas were classified by the World Health Organization (WHO) in 2007, including astrocytic tumors, oligoastrocytic tumors, oligodendrog- 
A

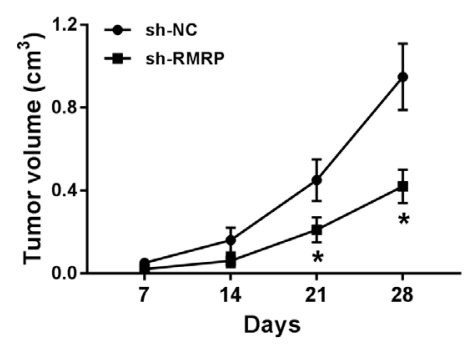

D

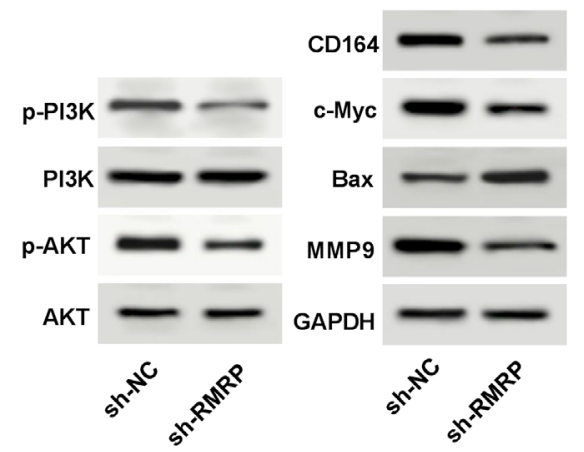

B

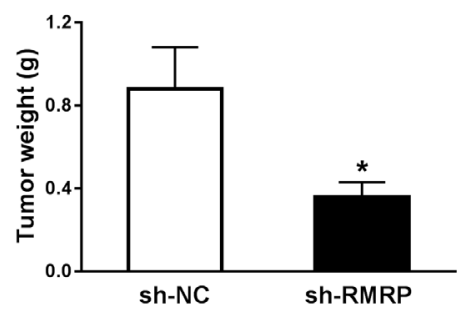

C

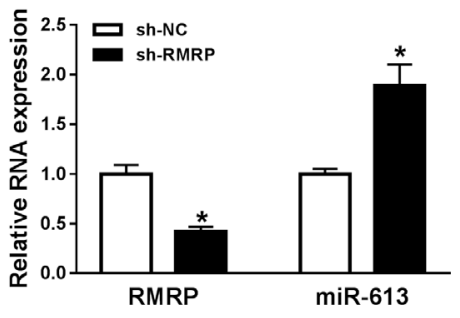

Figure 7. The knockdown of LINC00152 repressed the process of tumors in vivo. A) The tumor volumes were measured every 7 days, for 4 times, and the acquired data were analyzed. B) The tumor weights were measured after mice were sacrificed on day 28 . C) qRT-PCR was implemented to detect the expression of LINC00152 and miR-613 in tumor tissues. D) PI3K, p-PI3K AKT, p-AKT, CD164, c-Myc, Bax, and MMP9 were identified by western blot. ${ }^{*} \mathrm{p}<0.05$

lial tumors, ependymal tumors and neuronal and mixed neuronal-glial tumors [23]. More and more researches suggested that the pathophysiology of glioma was a complex process [24] and the regulatory mechanism needs further studies. Our study aimed to investigate the biological role of LINC00152 in the progression of glioma.

LncRNAs have been proved to participate in tumorigenesis. Overexpression of HOX-Antisense Intergenic RNA (HOTAIR) was associated with the progression of gastric cancer [25] and nuclear enriched abundant transcript 1 (NEAT1) was a fatal regulator of prostate cancer [26]. LINC00152 has been proved as an oncogene in tumor [9] and induced cell proliferation, migration, and invasion in bladder cancer by sponging microRNA-206 [27] and in non-small-cell lung cancer via regulating microRNA-1-3p [28]. Our study suggested that LINC00152 was upregulated in glioma tissues compared with normal para-cancer brain tissues; it was to say that LINC00152 took part in the development of glioma. What's more, LINC00152 acted as a biomarker in hepatocellular carcinoma clinical diagnosis [29]. It also viability and promotes apoptosis after downregulated by microRNA-376c-3p [30]. All evidences revealed that LINC00152 might play an utter role in the pathogenesis of human glioma; the conclusion was also verified by high expression of LINC00152 in glioma tissues.
Over the past decades, studies suggested that miRNA was extremely important in tumorigenesis, for example, microRNA-1 and microRNA-200 regulated tumorigenesis via Slug-independent mechanisms [31]; microRNA-1 blocked tumor proliferation and metastasis in nasopharyngeal carcinoma by targeting K-ras [32]. What's more, miR-613 hindered cell growth and invasion in human hepatocellular carcinoma by targeting doublecortin-like kinase 1 (DCLK1) [33] and functioned as a novel prognostic biomarker for human ovarian cancer [34]. Bioinformatics analysis manifested that miR-613 was a direct target gene of LINC00152, indicating that LINC00152 may regulate the progression of glioma through miR-613.

CD164 has been identified as a target gene of miR-613 and participated in the process of multiple tumors. CD164 modified the tumor formation of ovarian surface epithelial cells [35] and regulated tumor metastasis of prostate cancer though through the Stromal-Cell Derived Factor-1/ CXC chemokine receptor 4 (SDF-1a/CXCR4) axis [36]. Meanwhile, CD164 has also been proved to regulate cell proliferation and apoptosis in glioma by targeting phosphatase and tensin homolog deleted from chromosome 10 (PTEN) [37]. However, the potential molecular mechanism of CD164 needs further investigation. Emerging findings presented that a series of pathophysiological processes were 
regulated via PI3K signaling pathway, AKT was a downstream mediator of PI3K-dependent signaling [38]. We speculated that CD164 might regulate the process of glioma through the PI3K/AKT signaling pathway. The study analyzed the relative protein levels of PI3K/AKT pathway and the results verified the above conjecture.

Here, our study investigated the potential role of LINC00152 in the progression of glioma and found that LINC00152 regulated cell proliferation, apoptosis, migration, and invasion through the LINC00152/miR-613/CD164 axis via the PI3K/AKT signaling pathway in glioma. However, glioma tissues and nude mice experiments are required to further investigate these processes in glioma.

In conclusion, the levels of LINC00152 and CD164 were significantly repressed while miR-613 was obviously increased in glioma tissue samples and cell lines compared with relative control. What's more, miR-613 was a target gene of LINC00152 and directly targeted CD164. Knockdown of LINC00152 or CD164 induced cell apoptosis, repressed proliferation, migration and invasion in vitro. Additionally, miR-613 restored the effect of LINC00152 on cell proliferation, apoptosis, migration and invasion. The LINC00152/ miR-613/CD164 axis implicated in glioma via PI3K/AKT signaling pathway.

Supplementary information is available in the online version of the paper.

\section{References}

[1] WEN PY, KESARI S. Malignant Gliomas in Adults. N Engl J Med 2008; 359: 492-507. https://doi.org/10.1056/NEJMra0708126

[2] SANAI N, ALVAREZ-BUYLlA A, BERGER MS. Neural Stem Cells and the Origin of Gliomas. N Engl J Med 2005; 353: 811-822. https://doi.org/10.1056/NEJMra043666

[3] NAKAGAWA T, ENDO H, YOKOYAMA M, ABE J, TAMAI $\mathrm{K}$ et al. Large noncoding RNA HOTAIR enhances aggressive biological behavior and is associated with short disease-free survival in human non-small cell lung cancer. Biochem Biophys Res Commun 2013; 436: 319-324. https:// doi.org/10.1016/j.bbrc.2013.05.101

[4] YANG G, LU X, YUAN L. LncRNA: A link between RNA and cancer. Biochim Biophys Acta 2014; 1839: 1097-1109. https://doi.org/10.1016/j.bbagrm.2014.08.012

[5] ZHANG X, SUN S, PU JK, TSANG AC, LEE D et al. Long non-coding RNA expression profiles predict clinical phenotypes in glioma. Neurobiol Dis 2012; 48: 1-8. https://doi. org/10.1016/j.nbd.2012.06.004

[6] ZHENG HT, SHI DB, WANG YW, LI XX, XU Y et al. High expression of lncRNA MALAT1 suggests a biomarker of poor prognosis in colorectal cancer. Int J Clin Exp Pathol 2014; 7: 3174-3181.

[7] LI J, ZHANG M, AN G, MA Q. LncRNA TUG1 acts as a tumor suppressor in human glioma by promoting cell apoptosis. Exp Biol Med (Maywood) 2016; 241: 644-649. https:// doi.org/10.1177/1535370215622708
[8] Yunliang W, Yutong W, Jinfeng L, Yuzhen Z, Honglei Y, Bing H. CRNDE, a long-noncoding RNA, promotes glioma cell growth and invasion through mTOR signaling. Cancer Letters 2015 ; 367: 122-8.

[9] MENG Q, REN M, LI Y, SONG X. LncRNA-RMRP Acts as an Oncogene in Lung Cancer. PLoS One 2016; 11: e0164845. https://doi.org/10.1371/journal.pone.0164845

[10] FENG W, LI L, XU X, JIAO Y, DU W. Up-regulation of the long non-coding RNA RMRP contributes to glioma progression and promotes glioma cell proliferation and invasion. Arch Med Sci 2017; 13: 1315-1321. https://doi.org/10.5114/ aoms.2017.66747

[11] ZHU Z, DAI J, LIAO Y, MA J, ZHOU W. Knockdown of Long Noncoding RNA LINC00152 Suppresses Cellular Proliferation and Invasion in Glioma Cells by Regulating miR4775. Oncol Res 2018; 26: 857-867. https://doi.org/10.3727/ $096504017 X 15016337254597$

[12] LIU X, YIDAYITULA Y, ZHAO H, LUO Y, MA X et al. LncRNA LINC00152 promoted glioblastoma progression through targeting the miR-107 expression. Environ Sci Pollut Res Int 2018; 25: 17674-17681. https://doi.org/10.1007/ s11356-018-1784-x

[13] PEREIRA DM, RODRIGUES PM, BORRALHO PM, RODRIGUES CM. Delivering the promise of miRNA cancer therapeutics. Drug Discov Today 2013; 18: 282-289. https:// doi.org/10.1016/j.drudis.2012.10.002

[14] JAY C, NEMUNAITIS J, CHEN P, FULGHAM P, TONG AW. miRNA Profiling for Diagnosis and Prognosis of $\mathrm{Hu}-$ man Cancer. DNA Cell Biol. 2007 May; 26(5): 293-300. https://doi.org/10.1089/dna.2006.0554

[15] SHIMONO Y, ZABALA M, CHO RW, LOBO N, DALERBA $P$ et al. Downregulation of miRNA-200c links breast cancer stem cells with normal stem cells. Cell 2009; 138: 592-603. https://doi.org/10.1016/j.cell.2009.07.011

[16] LI X, ZHANG Y, ZHANG H, LIU X, GONG T et al. miRNA-223 Promotes Gastric Cancer Invasion and Metastasis by Targeting Tumor Suppressor EPB41L3. Mol Cancer Res 2011; 9: 824-833. https://doi.org/10.1158/1541-7786.MCR10-0529

[17] WANG Y, ZHANG X, LI H, YU J, REN X. The role of miRNA-29 family in cancer. Eur J Cell Biol 2013; 92: 123-128. https://doi.org/10.1016/j.ejcb.2012.11.004

[18] YU H, DUAN P, ZHU H, RAO D. miR-613 inhibits bladder cancer proliferation and migration through targeting SphK1. Am J Transl Res 2017; 9: 1213-1221.

[19] LI D, LI DQ, LIU D, TANG XJ. MiR-613 induces cell cycle arrest by targeting CDK4 in non-small cell lung cancer. Cell Oncol (Dordr) 2016; 39: 139-147. https://doi.org/10.1007/ s13402-015-0262-4

[20] FORDE S, TYE BJ, NEWEY SE, ROUBELAKIS M, SMYTHE J et al. Endolyn (CD164) modulates the CXCL12mediated migration of umbilical cord blood CD133+ cells. Blood 2007; 109: 1825-1833.

[21] TANG J, ZHANG L, SHE X, ZHOU G, YU F et al. Inhibiting CD164 expression in colon cancer cell line HCT116 leads to reduced cancer cell proliferation, mobility, and metastasis in vitro and in vivo. Cancer Invest 2012; 30: 380-389. https:// doi.org/10.3109/07357907.2012.666692 
[22] OSTROM QT, GITTLEMAN H, FARAH P, ONDRACEK A, CHEN Y et al. CBTRUS statistical report: Primary brain and central nervous system tumors diagnosed in the United States in 2006-2010. Neuro Oncol 2013; 15: ii1-56. https:// doi.org/10.1093/neuonc/not151

[23] LOUIS DN, OHGAKI H, WIESTLER OD, CAVENEE WK et al. The 2007 WHO Classification of Tumours of the Central Nervous System. Acta Neuropathol 2007; 114: 97-109. https://doi.org/10.1007/s00401-007-0243-4

[24] FURNARI FB, FENTON T, BACHOO RM, MUKASA A, STOMMEL JM et al. Malignant astrocytic glioma: genetics, biology, and paths to treatment. Genes Dev 2007; 21 : 26832710. https://doi.org/10.1101/gad.1596707

[25] ENDO H, SHIROKI T, NAKAGAWA T, YOKOYAMA M, TAMAI $\mathrm{K}$ et al. Enhanced expression of long non-coding RNA HOTAIR is associated with the development of gastric cancer. PLoS One 2013; 8: e77070. https://doi.org/10.1371/ journal.pone.0077070

[26] CHAKRAVARTY D, SBONER A, NAIR SS, GIANNOPOULOU E, LI R et al. The oestrogen receptor alpha-regulated lncRNA NEAT1 is a critical modulator of prostate cancer. Nat Commun 2014; 5: 5383. https://doi.org/10.1038/ncomms6383

[27] CAO HL, LIU ZJ, HUANG PL, YUE YL, XI JN. IncRNARMRP promotes proliferation, migration and invasion of bladder cancer via miR-206. Eur Rev Med Pharmacol Sci 2019; 23: 1012-1021. https://doi.org/10.26355/eurrev_201902_16988

[28] WANG Y, LUO X, LIU Y, HAN G, SUN D. Long noncoding RNA RMRP promotes proliferation and invasion via targeting miR-1-3p in non-small-cell lung cancer. J Cell Biochem 2019; 120: 15170-15181. https://doi.org/10.1002/jcb.28779

[29] LI J, WANG X, TANG J, JIANG R, ZHANG W et al. HULC and Linc00152 act as novel biomarkers in predicting diagnosis of hepatocellular carcinoma. Cell Physiol Biochem 2015; 37: 687-696. https://doi.org/10.1159/000430387
[30] ZHANG YH, FU J, ZHANG ZJ, GE CC, YI Y. LncRNALINC00152 down-regulated by miR-376c-3p restricts viability and promotes apoptosis of colorectal cancer cells. Am J Transl Res 2016; 8: 5286-5297.

[31] LIU YN, YIN JJ, ABOU-KHEIR W, HYNES PG, CASEY $\mathrm{OM}$ et al. MiR-1 and miR-200 inhibit EMT via Slug-dependent and tumorigenesis via Slug-independent mechanisms. Oncogene 2013; 32: 296-306. https://doi.org/10.1038/ onc. 2012.58

[32] CHEN X, SHI J, ZHONG J, HUANG Z, LUO X et al. miR-1, regulated by LMP1, suppresses tumour growth and metastasis by targeting K-ras in nasopharyngeal carcinoma. Int J Exp Pathol 2015; 96: 427-432. https://doi.org/10.1111/iep.12162

[33] WANG W, ZHANG H, WANG L, ZHANG S, TANG M. miR-613 inhibits the growth and invasiveness of human hepatocellular carcinoma via targeting DCLK1. Biochem Biophys Res Commun 2016; 473: 987-992. https://doi. org/10.1016/j.bbrc.2016.04.003

[34] ZHANG X, ZHANG H. Diminished miR-613 expression as a novel prognostic biomarker for human ovarian cancer. Eur Rev Med Pharmacol Sci 2016; 20: 837-841.

[35] HUANG AF, CHEN MW, HUANG SM, KAO CL, LAI HC et al. CD164 regulates the tumorigenesis of ovarian surface epithelial cells through the SDF-1a/CXCR4 axis. Mol Cancer 2013; 12: 115. https://doi.org/10.1186/1476-4598-12-115

[36] HAVENS AM, JUNG Y, SUN YX, WANG J, SHAH RB et al. The role of sialomucin CD164 (MGC-24v or endolyn) in prostate cancer metastasis. BMC Cancer 2006; 6: 195. https:// doi.org/10.1186/1471-2407-6-195

[37] TU M, CAI L, ZHENG W, SU Z, CHEN Y et al. CD164 regulates proliferation and apoptosis by targeting PTEN in human glioma. Mol Med Rep 2017; 15: 1713-1721. https:// doi.org/10.3892/mmr.2017.6204

[38] BRUHN MA, PEARSON RB, HANNAN RD, SHEPPARD KE. Second AKT: the rise of SGK in cancer signalling. Growth Factors 2010; 28: 394-408. https://doi.org/10.3109/ 08977194.2010 .518616 
Long non-coding RNA LINC00152/miR-613/CD164 axis regulates cell proliferation, apoptosis, migration and invasion in glioma via PI3K/AKT pathway

L. $\mathrm{ZHANG}^{1, *}, \mathrm{Y} . \mathrm{WANG}^{2}, \mathrm{H} . \mathrm{SU}^{2}$

Supplementary Information

A

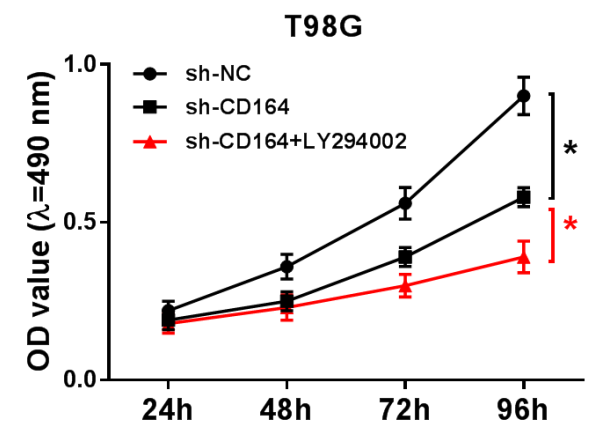

B

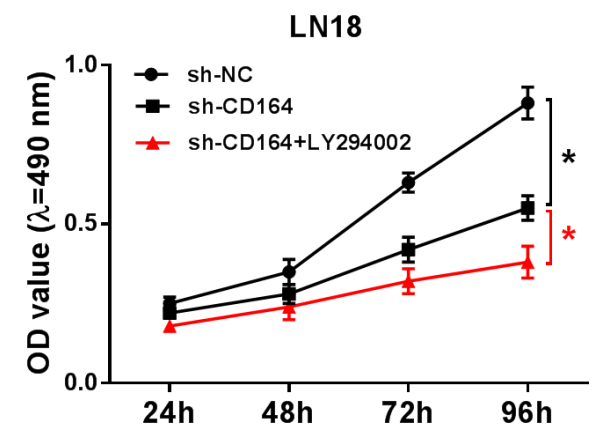

Supplementary figure S1. A, B) The role of sh-CD164 and PI3K inhibitor in cell proliferation was evaluated by MTT assay. ${ }^{*}<<0.05$ 